\title{
População quilombola e o câncer de colo uterino: uma revisão integrativa da literatura
}

\author{
Quilombola population and cervical cancer: an integrative literature review \\ Población de quilombola y cáncer de cuello uterino: una revisión integradora de la \\ literatura
}

Thamilly Joaquina Picanço da Silva ${ }^{1 *}$, Wingred Lobato Gonçalves ${ }^{1}$, Karoline Sampaio da Silva ${ }^{1}$, Luzilena de Sousa Prudêncio ${ }^{1}$, Rosana Oliveira do Nascimento ${ }^{1}$, Marlucilena Pinheiro da Silva1.

\section{RESUMO}

Objetivo: Analisar a relação entre o câncer de colo uterino e a população quilombola. Métodos: Trata-se de uma revisão integrativa da literatura, realizada na base de dados da Biblioteca Virtual de Saúde (BVS), utilizando-se os descritores, de acordo com os descritores em ciências da saúde (DeCS): "Neoplasias do colo uterino" e "Grupo de Ancestrais do Continente Africano", combinados pelo uso do operador Booleano AND. Os critérios de inclusão foram publicações dos últimos 5 anos em inglês, português e espanhol e excluiu-se os trabalhos repetidos e aqueles que não respondiam à pergunta de pesquisa proposta. Resultados: Por fim, selecionaram-se quatro artigos científicos para análise, os quais abordaram a relação entre a temática sob óticas diferentes, mas que conseguiam convergir para três principais temas: baixo conhecimento sobre 0 câncer de colo uterino entre as mulheres quilombolas, baixa adesão ao exame preventivo e etnoenfermagem, sabendo que o cuidado em saúde é um fator importante para esta população. Considerações finais: Os estudos selecionados para esta revisão mostraram a existência de relação entre o câncer de colo uterino e a população do estudo, identificando incipientes conhecimentos de quilombolas, sobre o risco de adquirirem uma doença como o câncer do colo do útero, as consequências da doença e a importância da prevenção.

Palavras-chave: Neoplasias do colo do útero, Saúde da mulher, Grupo de ancestralidade do continente africano.

\begin{abstract}
Objective: To analyze the relationship between cervical cancer and the quilombola population. Methods: It is an integrative literature review, carried out in the database of the Virtual Health Library (VHL), using the descriptors, according to the descriptors in health sciences (DeCS): "Cervical neoplasms "And" African Continental Ancestry Group ", combined using the Boolean AND operator. The inclusion criteria were publications from the last 5 years in English, Portuguese and Spanish, and repeated works and those that did not answer the proposed research question were excluded. Results: Finally, four scientific articles were selected for analysis, which addressed the relationship between the theme from different perspectives, but which managed to converge on three main themes: low knowledge about cervical cancer among quilombola women, low adherence preventive examination and ethno-nursing, knowing that health care is an important factor for this population. Final considerations: The studies selected for this review showed the existence of
\end{abstract}

1Universidade Federal do Amapá, Macapá - AM. `E-mail: thamillyps@gmail.com 
a relationship between cervical cancer and the study population, identifying incipient knowledge of quilombolas, about the risk of acquiring a disease such as cervical cancer, the consequences of disease and the importance of prevention.

Keywords: Cervical neoplasms, Women's health, African continental ancestry group.

\section{RESUMEN}

Objetivo: Analizar la relación entre el cáncer de cuello uterino y la población quilombola. Métodos: Es una revisión integradora de la literatura, realizada en la base de datos de la Biblioteca Virtual en Salud (BVS), utilizando los descriptores, según los descriptores en ciencias de la salud (DeCS): "Neoplasias cervicales" y "Grupo de ascendencia continental africana", combinado usando el operador booleano AND. Los criterios de inclusión fueron publicaciones de los últimos 5 años en inglés, portugués y español, y se excluyeron trabajos repetidos y que no respondieran a la pregunta de investigación propuesta. Resultados: Finalmente, se seleccionaron cuatro artículos científicos para el análisis, los cuales abordaron la relación entre el tema desde diferentes perspectivas, pero que lograron converger en tres grandes ejes: bajo conocimiento sobre el cáncer de cuello uterino entre las mujeres quilombolas, examen preventivo de baja adherencia y etno-enfermería, sabiendo que la atención de la salud es un factor importante para esta población. Consideraciones finales: Los estudios seleccionados para esta revisión evidenciaron la existencia de relación entre el cáncer de cuello uterino y la población de estudio, identificando conocimientos incipientes de los quilombolas, sobre el riesgo de adquirir una enfermedad como el cáncer de cuello uterino, las consecuencias de la enfermedad y la importancia de la prevención.

Palabras clave: Neoplasias cervicales, salud de la mujer, grupo de ascendencia continental africana.

\section{INTRODUÇÃO}

O câncer de Colo Uterino (CCU) é uma doença de natureza crônica, com origem em alterações intraepiteliais que podem se transformar em um processo invasor. É caracterizado pela replicação desordenada do epitélio de revestimento do colo, comprometendo o estroma (tecido subjacente) e invadindo estruturas e/ou órgãos podendo eles serem próximos ou a distância. Segundo a World Health Organization (WHO) (2014) existem duas principais categorias de carcinomas que podem agredir o do colo do útero: o carcinoma epidermoide e o adenocarcinoma, dentre eles aquele que mais ocorre é do tipo epidermoide, sendo responsável por mais de $80 \%$ dos casos aproximadamente, enquanto que o adenocarcinoma é ocorre mais raramente e em menos de $10 \%$ do total de casos.

O desenvolvimento desta doença é lento e assintomático principalmente no estágio inicial, a fisiopatologia segundo Brasil (2011) inclui "quadros de sangramento vaginal intermitente ou após a relação sexual, secreção vaginal anormal e dor abdominal associada com queixas urinárias ou intestinais nos casos mais avançados".

No Brasil, esta neoplasia é considerada um dos mais preocupantes e graves problemas de saúde pública, acometendo mulheres na faixa etária compreendida entre 35 e 50 anos, em plena idade reprodutiva. A evolução da doença é lenta e passa por fases pré-clínicas (benignas) tendo como características lesões que antecedem a instalação das células cancerosas, tais manifestações quando detectadas precocemente tem grandes chances de cura. Dessa forma, tendo um alto grau de prevenção de um quadro maligno, quando diagnosticado previamente, no entanto o número de mulheres que morrem acometidas por esta doença tem aumentado na última década (TSUCHIYA C, et al., 2017).

Duarte GS (2017) afirma que dados de mortalidade no Brasil, mostram que o câncer de colo uterino, junto com câncer de mama, é a principal causa de morte em mulheres com menos de 50 anos de idade. Segundo o autor, mesmo com todos os programas de prevenção, existem falhas consideráveis na adesão das medidas, 
que apontaram as dificuldades nas estratégias de prevenção como sendo o fator primário para 0 desenvolvimento da neoplasia. Alguns fatores e comportamentos de risco estão associados ao desenvolvimento e progressão do câncer de colo do útero, dentre eles à presença de infecção associada com o Papilomavírus Humano (HPV) identificado como o principal fator etiológico da neoplasia do colo de útero, a partir da década de 70 .

Aproximadamente 118 tipos de Papiloma Vírus Humano foram completamente descritos e cerca de 100 tipos que acometem o ser humano já foram identificados, sendo que os tipos 16 e 18 como os mais malignos para o desenvolvimento de CCU (SCHIER J, et al., 2003).

A incidência do Carcinoma Cérvico uterino e a mortalidade decorrente da sua evolução estão fortemente associadas às precárias condições socioeconômicas dos países em desenvolvimento. Dentre os fatores de risco é possível destacar o tabagismo como fator que favorece o aumento do risco para o acometimento pela doença. O fumo pode causar uma imunossupressão local, que permite com maior facilidade a penetração do vírus nas células, além disto, o epitélio vaginal das fumantes tem menos células de Langerhans, facilitando as infecções virais e o aparecimento da neoplasia (MATTA F, 2011).

Existem ainda fatores relacionados à saúde sexual e reprodutiva das mulheres tais como, o início precoce da atividade sexual, o qual pode promover maior risco de transformação neoplásica no colo do útero na presença do HPV, pois quanto maior a incidência desse vírus nesta fase da vida, existe um grande risco de câncer no futuro. A multiplicidade de parceiros sexuais e a promiscuidade também são fatores de risco importantes para a infecção do Papiloma Vírus Humano e por isso favorecem o desenvolvimento da neoplasia de câncer de colo uterino (LIMA RE, et al., 2013).

Outro fator de risco muito expressivo dentro da literatura é a falta de informação e desconhecimento sobre a realização do exame preventivo do câncer de colo uterino, muitas mulheres não buscam o exame por tais motivos, demonstrando a necessidade de investimentos em trabalhos de promoção à saúde, onde a prevenção do câncer cervical deveria atentar para ações como a "participação informada", por parte das mulheres, no rastreamento, reconhecendo os seus valores e práticas e os utilizando como meio de multiplicidade de informação (SOUZA AF e COSTA LHR, 2015).

De acordo com a Organização Mundial da Saúde (OMS), as estratégias para a detecção são o diagnóstico precoce (abordagem de pessoas com sinais e/ou sintomas da doença) e o rastreamento (aplicação de um teste ou exame numa população assintomática, aparentemente saudável, com objetivo de identificar lesões sugestivas de câncer e encaminhá-la para investigação e tratamento) (WORLD HEALTH ORGANIZATION, 2007). A prevenção do câncer invasivo do colo do útero é feita por medidas educativas, vacinação, rastreamento, diagnóstico e tratamento das lesões subclínicas.

O exame preventivo do câncer do colo do útero (Papanicolau) é a principal estratégia para detectar lesões precursoras e fazer o diagnóstico da doença, no entanto, percebe-se que ainda hoje se encontra dificuldades na promoção à saúde de populações marginalizadas, como as comunidades quilombolas. O sistema de saúde tem lacunas quanto às políticas públicas voltada para as comunidades quilombolas, que apresentam precariedade na oferta dos serviços de saúde, por fatores como distância dos centros urbanos, falta de profissionais capacitados para atuação com a comunidade, ou a dificuldade em aceitação na implantação dos serviços por parte da população (SILVA A 2017).

Aproximadamente $40 \%$ das mulheres brasileiras nunca foram submetidas ao exame preventivo de Papanicolaou e, apenas cerca de $7,7 \%$ das brasileiras são cobertas por programas governamentais de prevenção e controle do câncer de colo de útero pela realização do exame citopatológico, sendo que há deficiência na adesão entre as mulheres negras, que são majoritariamente a maior parcela do país, ressaltando as negras quilombolas (FERNANDES RAQ e NARCHI NZ, 2002; ZANOTELLI T, 2013; JACINTHO KS, et al., 2018).

Dessa forma, o cuidado à saúde é um fator importante para os quilombolas, que mesmo com o processo de enculturação vivenciado, ainda é modificado e moldado pela cultura deste povo, que tradicionalmente envolve valores, costumes, crenças e tradições, auxiliando na manutenção de seu bem-estar, assim como no 
enfrentamento de doenças, incapacidades ou da morte (REIS A, et al., 2012). Diante do exposto é necessário que haja um investimento na formação e qualificação dos profissionais de saúde que atuam em comunidades quilombolas acerca da importância de se praticar de forma efetiva as políticas públicas de saúde voltadas para este grupo populacional.

Para tanto, é imperativo que se desenvolva pesquisas voltadas para a investigação das questões que envolvem a realidade social e de saúde, e dessa forma trazer respostas aos questionamentos que permeiam a saúde dessa população, a qual é foco deste estudo. Com o intuito de correlacionar a relevância científica e a efetivação das políticas públicas de saúde típicas da comunidade quilombola, apresentamos que o objetivo deste estudo foi analisar a produção científica acerca da relação entre o câncer de colo uterino e a população quilombola publicada nos últimos 5 anos.

\section{MÉTODOS}

Revisão integrativa da literatura, de abordagem qualitativa, descritiva e exploratória, realizada em maio de 2020 e norteada pela seguinte questão de pesquisa: o que dizem as evidências científicas sobre a relação entre o câncer de colo uterino e a população quilombola? Na elaboração deste artigo se obedeceu às etapas a seguir para uma maior exatidão e confiabilidade do estudo: Identificação do tema de afinidade; Elaboração da questão de pesquisa; Estabelecimento dos Descritores de saúde; Definição dos critérios de inclusão e exclusão de estudos; Determinação dos pontos principais a serem extraídos das publicações científicas; Avaliação e interpretação dos estudos.

A busca ocorreu na BVS, a qual é o espaço virtual de interação, informação e cooperação técnica, cujo o objetivo principal é permitir um maior acesso a comunidade cientifica da América Latina e Caribe, promovendo a descentralização do conhecimento e democratização as fontes de informação; tal plataforma está disponível em três idiomas (inglês, português e espanhol) e possibilita uma busca avançada com a utilização de filtros e exportação dos resultados. Para a busca utilizou-se os Descritores em Ciências da Saúde (DeCS) "Neoplasias do colo do uterino" e "Grupo de Ancestralidade do Continente Africano", combinados pelo operador booleano "AND". A associação dos descritores permitiu capturar 204 trabalhos, os quais foram filtrados pelos seguintes critérios de inclusão: texto completo, em inglês, português e espanhol nos últimos cinco anos.

Os critérios de exclusão foram trabalhos duplicados, resumos de eventos anais, dissertações, teses, artigos que não se enquadram no recorte temporal ou que fogem do tema e consequentemente não atendem a temática proposta, desse modo que não respondiam à questão de pesquisa apresentada neste estudo. Foram realizadas leitura e análise dos resumos dos trabalhos capturados, ao final foram selecionadas quatro publicações científicas para compor a amostra da pesquisa, pois os textos versavam argumentação lógica, crítica teórico-metodológica e síntese conclusiva sobre o tema abordado.

Por não se tratar de pesquisa aplicada, não foi necessária submissão e aprovação por Comitê de Ética em Pesquisa. Refere-se, contudo, que todos os autores consultados foram devidamente citados e referenciados, de forma a respeitar as recomendações éticas contidas na Lei número 9.610, de 19 de fevereiro de 1998, que regulamenta os direitos autorais (BRASIL, 1998).

\section{RESULTADOS}

Foram capturadas 204 publicações científicas, que tiveram o seu aceite entre os anos de 2015 e 2020 . Pela combinação dos descritores de saúde apresentados na base de dados, após aplicar os critérios de inclusão, foi possível eleger 22 artigos para a pré-seleção, após a leitura atenta e minuciosa dos títulos e resumos.

A partir da análise destes trabalhos, 18 foram excluídos: quatro porque estavam repetidos e 14 porque não respondiam à pergunta de pesquisa. Diante disso, foram selecionados para a amostra final deste estudo quatro artigos, descrevendo os pontos mais importantes para a apreciação coerente quanto ao tema definido (Quadro 1). 
Quadro 1 - Mapeamento da literatura selecionada para análise, publicada entre 2015 e 2020.

\begin{tabular}{|c|c|c|c|c|c|}
\hline ID & AUTORES/ANO & PERIÓDICO & $\begin{array}{l}\text { OBJETIVO } \\
\text { GERAL }\end{array}$ & $\begin{array}{c}\text { TIPO DE } \\
\text { ESTUDO/LOCAL }\end{array}$ & CONCLUSÃO \\
\hline 1 & $\begin{array}{c}\text { RAMOS- } \\
\text { JARABA SM e } \\
\text { PINEDA MC. }\end{array}$ & $\begin{array}{l}\text { Universidad } \\
\text { y Salud }\end{array}$ & $\begin{array}{l}\text { Compreender os } \\
\text { significados sobre } \\
\text { Câncer de ama ou } \\
\text { colo uterino com os } \\
\text { quais as mulheres } \\
\text { afrodescendentes } \\
\text { cuidavam nos } \\
\text { serviços de saúde } \\
\text { de Medellín, } \\
\text { Colômbia. }\end{array}$ & $\begin{array}{c}\text { Pesquisa } \\
\text { qualitativa com } \\
\text { enfoque } \\
\text { etnográfico / } \\
\text { Colômbia }\end{array}$ & $\begin{array}{l}\text { A doença transpassou } \\
\text { o ser biológico, pois } \\
\text { alterou a vida social, } \\
\text { familiar e emocional } \\
\text { dos Afrodescendentes, } \\
\text { por seu papel ancestral } \\
\text { como cuidadores da } \\
\text { saúde familiar. }\end{array}$ \\
\hline 2 & $\begin{array}{l}\text { FERNANDES } \\
\text { ETBS, et al. }\end{array}$ & $\begin{array}{l}\text { Revista } \\
\text { Gaúcha } \\
\text { de } \\
\text { Enfermagem }\end{array}$ & $\begin{array}{l}\text { Discutir as práticas } \\
\text { de prevenção do } \\
\text { câncer do colo do } \\
\text { útero de mulheres } \\
\text { quilombolas. }\end{array}$ & $\begin{array}{c}\text { Estudo qualitativo } \\
\text { com } \\
\text { etnoenfermagem } \\
\text { / Brasil }\end{array}$ & $\begin{array}{l}\text { Questões de ordem } \\
\text { social, cultural e de } \\
\text { acesso relacionam-se } \\
\text { com as práticas } \\
\text { preventivas para o } \\
\text { câncer do colo uterino } \\
\text { de quilombolas. Assim, } \\
\text { torna-se imprescindível } \\
\text { um planejamento de } \\
\text { cuidados congruentes } \\
\text { com a realidade } \\
\text { dessas mulheres. }\end{array}$ \\
\hline 3 & $\begin{array}{l}\text { BOA SORTE } \\
\text { ET, et al. }\end{array}$ & $\begin{array}{c}\text { Revista } \\
\text { Baiana de } \\
\text { Enfermagem }\end{array}$ & $\begin{array}{c}\text { Descrever o } \\
\text { conhecimento de } \\
\text { mulheres } \\
\text { quilombolas sobre } \\
\text { o câncer de colo } \\
\text { uterino. }\end{array}$ & $\begin{array}{c}\text { Estudo } \\
\text { qualitativo/ Brasil }\end{array}$ & $\begin{array}{c}\text { Evidente } \\
\text { desconhecimento das } \\
\text { participantes sobre seu } \\
\text { próprio corpo e sobre a } \\
\text { etiopatogenia do } \\
\text { câncer de colo uterino, } \\
\text { mostrando a } \\
\text { necessidade } \\
\text { e a importância do } \\
\text { estabelecimento de } \\
\text { atividades educativas } \\
\text { participativas. }\end{array}$ \\
\hline 4 & $\begin{array}{l}\text { JACINTHO KS, } \\
\text { et al. }\end{array}$ & $\begin{array}{l}\text { Cultura de } \\
\text { los Cuidados }\end{array}$ & $\begin{array}{l}\text { Identificar os } \\
\text { fatores que } \\
\text { influenciam na } \\
\text { prevenção do } \\
\text { câncer cérvico- } \\
\text { uterino em } \\
\text { mulheres } \\
\text { quilombola. }\end{array}$ & $\begin{array}{c}\text { Pesquisa } \\
\text { qualitativa com } \\
\text { enfoque } \\
\text { etnográfico/ Brasil }\end{array}$ & $\begin{array}{l}\text { Falta de orientação } \\
\text { acerca da importância } \\
\text { do acompanhamento } \\
\text { ginecológico e da } \\
\text { realização anual do } \\
\text { exame citopatológico. }\end{array}$ \\
\hline
\end{tabular}

Fonte: Silva TJP, et al., 2021.

Em relação à base de dados da indexação, 1 estava na MEDLINE, 1 na IBECS e 2 na LILACS. Quanto ao país de origem dos estudos, 3 foram realizados no Brasil, localizados na região nordeste e apenas 1 foi realizado no exterior (Colômbia), demonstrando importância e ênfase para a necessidade desses estudos no país. Se tratando do idioma, 3 deles publicados em português e 1 em espanhol.

Sobre o tipo de estudo, os 4 foram pesquisas originais e do tipo qualitativo, sendo $2 \mathrm{com}$ abordagem etnográfica, $1 \mathrm{com}$ abordagem na etnoenfermagem e 1 abordando a oficina feminista. Apresentamos 0 organograma que ilustra a busca dos materiais (Figura 1). 
Figura 1 - Organograma da coleta de dados.

PERGUNTA DA PESQUISA: "O que dizem as evidências científicas sobre a relação entre o câncer de colo uterino e a população quilombola?".

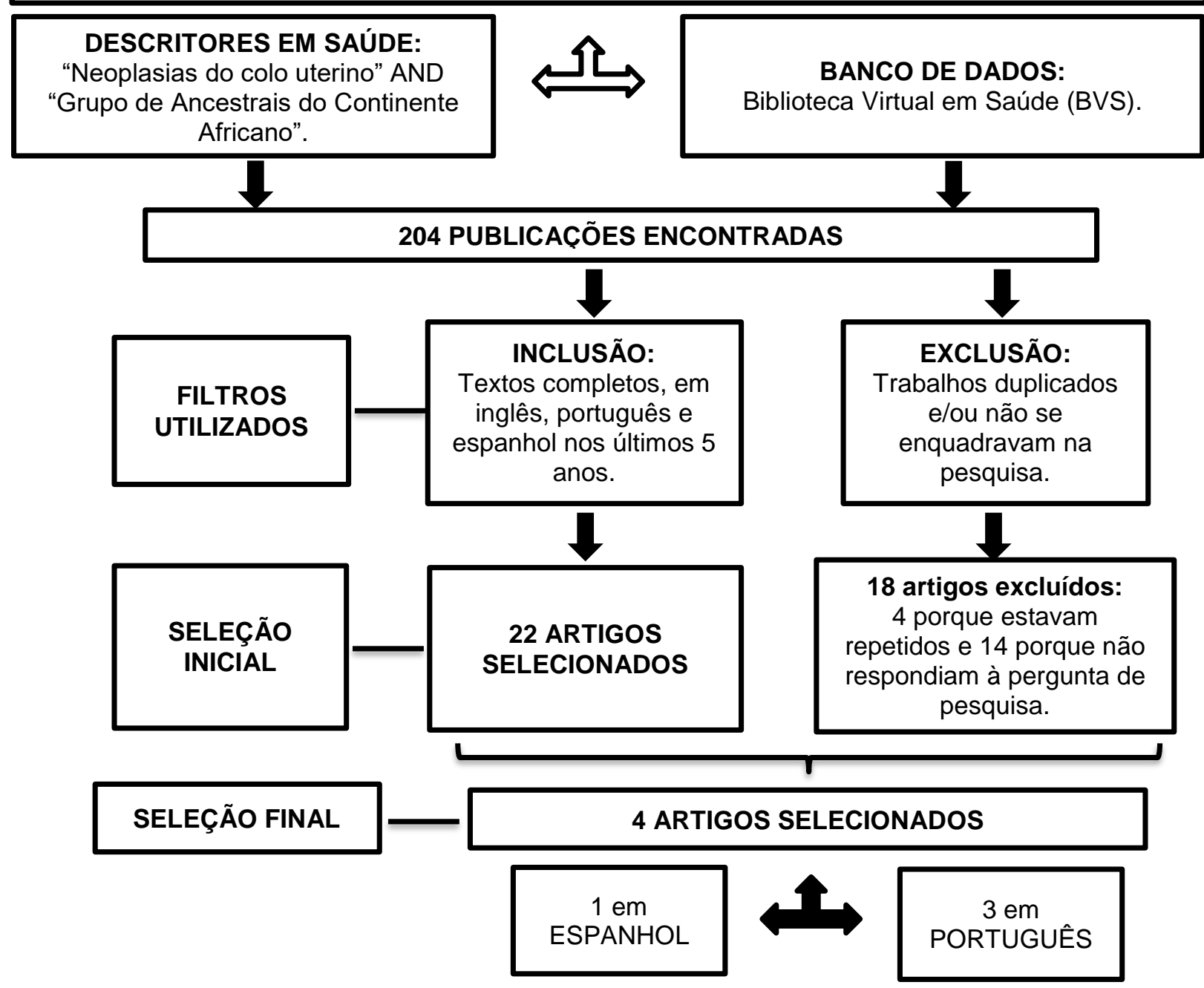

Fonte: Silva TJP, et al., 2021.

De acordo com Jacintho KS, et al. (2018) o nível de conhecimento sobre o CCU ainda é muito elementar entre as mulheres de comunidades quilombolas, tal ponto traz grandes consequências para esse povo, como à baixa conscientização quanto ao significado, a não sensibilização para a realização do exame preventivo, além de fatores estruturais como: o restrito acesso a assistência à saúde. Tais situações estão diretamente ligadas ao número de mulheres que nunca realizaram o exame de Papanicolau e que são diagnosticadas tardiamente com a doença.

Essa discussão concerne com o histórico dessas comunidades remanescentes quilombolas, tendo em vista que foram originadas decorrentes de um processo histórico iniciado com a colonização portuguesa, diante do avanço dos povos ocidentais sobre os índios e negros, estes tiveram que lutar contra a ideologia racista, a qual foi nutrida por uma concepção etnocentrista, que oprimiu estes povos, os deixando à margem de serviços sociais como a educação e a saúde (JORDANA L e VOGADO P, 2014; FERNANDES SL e SANTOS AO, 2016).

De acordo com o estudo de Oliveira MV, et al. (2014) as comunidades quilombolas se destacam e se inserem de modo exacerbado nas situações de desigualdades sociais, justamente por sua descendência e ancestralidade negra. Desse modo ainda segundo os autores, tal população pode ter um impacto direto nos 
seus indicadores de saúde, por conta das mais diversas dificuldades do sistema de saúde, que chega com força ainda maior dentro do contexto quilombola, onde se ressalta a vulnerabilidade social, expropriação cultura e a diminuição de direitos. Sabe-se ainda que em função da construção da mátria brasileira, os quilombolas passaram por diversos processos culturais e comunitários, no entanto, ainda permanecem em um isolamento físico e social, que repercutem em desigualdades visíveis no contexto de saúde.

Diante disso, as manifestações culturais as quais se perpetuaram durante essa edificação histórica, influenciam o seu cotidiano e, sobretudo, o processo de saúde e doença (SILVA MJ, et al., 2010; PRATES LA, 2015). Dessa forma, o cuidado à saúde é um fator importante para os quilombolas, e mesmo com o processo de enculturação vivenciado, ainda é modificado e moldado pela cultura deste povo, que tradicionalmente envolve valores, costumes, crenças e tradições, auxiliando na manutenção de seu bemestar, assim como no enfrentamento de doenças, incapacidades ou da morte (REIS A, et al., 2012).

Para os autores Ramos-Jaraba SM e Pineda MC (2018) as mulheres quilombolas construíram o significado do câncer além do ser biológico, trazem consigo significados que representam uma alteração na vida social, familiar e emocional como resultado da doença, além de identificar a relação da enfermidade, com o espaço social e geográfico da região que eles vivem, que de uma maneira ou de outra as determina.

Tal achado supracitado é afirmado por Boa Sorte ET, et al. (2016) quando descreve que as mulheres quilombolas narram o CCU como uma doença fatal e perigosa, cujo tratamento traz muito sofrimento. Os autores ainda associam isso ao fato de que os cânceres de modo geral são vistos por toda a população como uma doença gravíssima e que tem alta taxa de letalidade. Ainda ressaltam que esta população pouco conhece o seu próprio corpo e a forma que a patologia se manifesta no organismo humano, bem como as dificuldades de entendimento sobre sua localização anatômica.

Fernandes ETBS, et al. (2018) tende a explicar tal fato ao associar que as pessoas negras, especialmente as mulheres, recebem os mais baixos salários, com baixo nível de escolaridade, expondo este grupo populacional às vulnerabilidades relacionadas ao acesso à informação em saúde e prevenção de doenças. Assim, estes fatores socioeconômicos geram desigualdades sobre a saúde das mulheres negras, as quais devem ser levadas em consideração na análise do processo saúde-doença desta população.

Ramos-Jaraba SM e Pineda MC (2018) discorrem que existe desigualdade no modelo de assistência médica, a qual se exacerba principalmente nas minorias étnicas, e não seria diferente nas populações negras quilombolas, destaca ainda que as opressões a que as mulheres são expostas, agravam sua situação econômica e social. Mostram ainda que acima de tudo estas precisam enfrentar desigualdades que envolvem até o espaço geográfico, pois muitas comunidades quilombolas são distantes dos grandes centros de saúde, o que dificulta o acesso de mulheres em busca de prevenção e tratamento para a doença.

Jacintho KS, et al. (2018) associa esses fatores à baixa adesão ao exame preventivo do câncer do colo, confirmou tal conjectura quando garantiu que os fatores relacionados ao pouco conhecimento das mulheres quilombolas sobre: importância da realização do exame preventivo do $\mathrm{CCU}$, fisiopatologia da doença, localização e quais os possíveis sintomas, ainda é um fator de grande relevância para que o índice desta doença continue a aumentar e oferecer riscos à sua saúde.

Tal evidência é corroborada pelo estudo de Pimentel AV, et al. (2011) no qual destaca que as mulheres quilombolas apesentaram uma carência significativa do conhecimento sobre os cuidados de prevenção do câncer de colo do útero e ainda destacou a recorrência do medo da doença, do medo do exame citopatológico, do medo do resultado do exame, dos diversos "medos" que cercam estas mulheres associados ainda a vergonha em buscar os cuidados, a dificuldade de se encontrar uma unidade de saúde receptiva e acolhedora.

Desse modo a prevenção por parte das mulheres quilombolas, seja por conta inacessibilidade do sistema ou pela falta de vontade da usuária, acarreta em um alcance muito pequeno em comparação a necessidade dessa população. Boa Sorte ET (2015) constatou no seu estudo que as tradições de ancestralidade africana ainda estão muito enraizadas principalmente nas mulheres de maior idade, se referindo ao uso de plantas, ervas e raízes como prevenção para doenças, entre elas que também incluem o câncer de colo uterino e as 
patologias que envolvem o sistema reprodutor. A autora destaca ainda que é preciso levar em consideração este cuidado genérico citado por diversas vezes entre as participantes do estudo, para que o conhecimento empírico e experenciado no cotidiano seja utilizado em favor da saúde das mesmas.

Fernandes ETBS, et al. (2018) utiliza a etnoenfermagem ou cuidado cultural como uma forma de auxiliar nestas mazelas sociais, levando em consideração os conhecimentos e práticas de cuidado local, tradicional e popular, transmitidos e aprendidos para assistir, dar suporte aos que necessitam de saúde a fim de melhorar o bem-estar. Os autores ainda destacam algumas das práticas tradicionais quilombolas, preservadas pelas mulheres mais velhas, que passam desde o tratar a inflamação e "quentura no útero" até o prevenir o câncer do colo do útero com plantas medicinais (barbatimão, romã, jatobá e quixaba),

A capacidade de cuidar da mulher é reconhecida no seu âmbito familiar, por suas habilidades e capacidades ligadas, à autoconfiança e à autonomia. Dessa forma o papel de cuidadora, deve ser visto como uma medida de promoção da saúde, pois dentro das comunidades tradicionais, existe a condição de gênero, de forma marcante no cotidiano (CARVALHAES MABL e BENÍCIO MHD, 2002).

É importante gerar maior visibilidade do processo saúde-doença das mulheres afrodescendentes, pois desempenham papel fundamental na visão de mundo da cultura afrodescendente, pois são elas que têm um papel ancestral como cuidadoras para a preservação das práticas de cuidados de saúde, delas próprias e da comunidade (RAMOS-JARABA SM e PINEDA MC, 2018).

\section{CONSIDERAÇÕES FINAIS}

Os estudos selecionados para esta revisão permitiram identificar que a relação entre o CCU e as mulheres quilombolas na literatura estão voltadas principalmente para a ausência ou incipiente conhecimento sobre o risco de adquirirem uma doença como o câncer do colo do útero, as consequências da doença e a importância da prevenção, destacando ainda diversos motivos (históricos, sociais, econômicos, estruturais e outros) para as mulheres não procurarem os serviços de saúde. Investir na qualificação dos trabalhadores de saúde para a atenção e cuidado aos usuários quilombolas e sua cultura é tarefa essencial e proposição das políticas públicas brasileiras, no entanto, os estudos aqui apresentados, revelam que ainda estamos distantes de tal feito. O estudo revela a necessidade emergencial de uma ação abrangente e planejada, que permita reduzir ou até mesmo anular, os efeitos acumulados da omissão do direito à saúde, em especial os Quilombolas, foco desse estudo.

\section{REFERÊNCIAS}

1. BOA SORTE ET, et al. Conocimiento de mujeres quilombolas sobre el cáncer del colo uterino. Revista Baiana de Enfermagem, 2016; 30(1): 325-334.

2. BOA SORTE ET. Práticas preventivas para o câncer do colo uterino: um estudo com mulheres quilombolas. Dissertação (Mestrado em Enfermagem) - Universidade Federal da Bahia, Salvador Maria, 2015.

3. BRASIL. Instituto Nacional do Câncer José Alencar Gomes da Silva. Programa Nacional de controle do câncer do colo de útero. Revista Brasileira de Cancerologia, 2011; 46(1): 1-14.

4. BRASIL. Presidência da República. Lei no 9.610, de 19 de fevereiro de 1998. Altera, atualiza e consolida a legislação sobre direitos autorais e dá outras providências. Brasília: Presidência da República, 1998.

5. CARVALHAES MABL, BENÍCIO MHD. Capacidade materna de cuidar e desnutrição infantil. Revista de Saúde Pública, 2002; 36(2): 188-197.

6. DUARTE GS. Plano de intervenção para diminuir o número de casos de câncer de colo de útero em vianópolis Betim -Minas Gerais. Trabalho de Conclusão de Curso. Minas Gerais, 2017; 0-33.

7. FERNANDES RAQ, NARCHI NZ. Knowledge of pregnant women on the tests to early detect cervix uteri and breast cancer in a poor community. Revista Brasileira de Cancerologia, 2002; 48(2):223-230.

8. FERNANDES ETBS, et al. Prevenção do câncer do colo uterino de quilombolas à luz da teoria de Leininger. Revista Gaúcha de Enfermagem, 2018; 39(1): e2016-4.

9. FERNANDES SL, SANTOS AO. Itinerários terapêuticos de mulheres quilombolas de Alagoas, Brasil. Interfaces Brasil/Canadá, 2016; 16(2): 127-143. 
10. JACINTHO KS, et al. Factores que influyen en la prevención del cáncer de cuello uterino en la comunidad quilombolas. Revista de Enfermería y Humanidades Cultura de los Cuidados, 2018; 22(50): 151-157.

11. JORDANA L, VOGADO P. Mulheres quilombolas: no rastro da interface saúde, raça e cultura. Trabalho de Conclusão de Curso (Graduação em Saúde Coletiva) - Universidade de Brasília, Brasília, 2014.

12. LIMA RE, et al. Conhecimento E Prática Na Realização Do Exame De Papanicolaou e Infecção por HPV em Adolescentes De Escola Pública. Revista Paraense de Medicina. 2013, 27(4):59-66.

13. MATTA F. O tabagismo e a oncogênese do câncer de colo uterino. Trabalho de Conclusão de Curso, 2011; 111127.

14. OLIVEIRA MV, et al. Fatores associados a não realização de Papanicolau em mulheres quilombolas. Ciência \& Saúde Coletiva, 2014; 19: 4535-4544.

15. PIMENTEL AV, et al. Percepção da vulnerabilidade entre mulheres com diagnóstico avançado do câncer do colo do útero. Texto \& Contexto-Enfermagem, 2011, 20(2): 255-262.

16. PRATES LA. O olhar feminino sobre o cuidado à saúde da mulher quilombola. Dissertação (Mestrado em Enfermagem) - Universidade Federal de Santa Maria, Santa Maria, 2015.

17. RAMOS-JARABA SM, PINEDA MC. Significados que construyen mujeres afrodescendientes frente al cáncer de mama y cuello uterino, atendidas en Medellín, Colombia. Universidad y Salud, 2018; 20(2): 111-120.

18. REIS $\mathrm{A}$, et al. $\mathrm{O}$ cuidado à mulher na contemporaneidade: reflexões teóricas para o exercício da enfermagem transcultural. Revista Mineira de Enfermagem, 2012; 16(1): 129-135.

19. SCHIER J, et al. Programa de acompanhante hospitalar para paciente geriátrico. Revista Gaúcha de Enfermagem, 2003; 24(1): 61-68.

20. SILVA A. Câncer do Colo do Útero: a importância do exame citológico na sua prevenção. Trabalho de Conclusão de Curso (Graduação em Enfermagem) - União Metropolitana de Educação e Cultura, Lauro de Freitas, 2017.

21. SILVA MJ, et al. Uso dos serviços Públicos de Saúde para DST/ HIV/Aids por comunidades remanescentes de quilombos no Brasil. Saúde \& Sociedade, 2010; 19(2): 109-120.

22. SOUZA AF, COSTA LHR. Conhecimento de Mulheres sobre HPV e Câncer do Colo do Útero após Consulta de Enfermagem. Cancerologia, 2015; 61(4): 343-350.

23. TSUCHIYA C. et al. O câncer de colo do útero no Brasil: uma retrospectiva sobre as políticas públicas voltadas à saúde da mulher. Jornal Brasileiro de Economia da Saúde, 2017; 9(1): 137-147.

24. WORLD HEALTH ORGANIZATION. Cancer control: knowledge into action. Geneva: WHO, 2007.

25. WORLD HEALTH ORGANIZATION. Comprehensive Cervical Cancer Control. Geneva: WHO, 2014.

26. ZANOTELLI T. A percepção de mulheres sobre o exame citopatológico. Trabalho de Conclusão de Curso. Lajeada, 2013; 0-33. 